\title{
FlexRay Protocol Controller Conformance Test Implementation
}

\author{
Chu Liu*, Feng Luo \\ Clean Energy Automotive Engineering Center, College of Automotive Engineering, Tongji University, Shanghai \\ 201804, China. \\ * Corresponding author. Tel: +86-21-69589482; email: liuchu1985@126.com \\ Manuscript submitted December 24, 2015; accepted June 14, 2016. \\ doi: 10.17706/ijcee.2016.8.3.250-258
}

\begin{abstract}
The paper proposes a new approach for testing FlexRay communication controller at protocol level The implementation is realized on the basis of FlexRay international standard ISO 17458-3, which goes beyond regular frame level testing that is provided by common FlexRay simulation and test tools. With the help of arbitrary sequence generator implemented in FPGA and test engine implemented on computer, the protocol related test cases such as static segment, dynamic segment, clock synchronization, wakeup and startup are fully supported. This design is verified by oscilloscope as according to the specification. Verification results show that the design and testing requirements are well satisfied.
\end{abstract}

Key words: FlexRay bus, automotive testing, automotive network, FPGA.

\section{Introduction}

FlexRay is an automotive network communications protocol initially developed by the FlexRay Consortium to govern on-board automotive computing. It is designed to be faster and more reliable than established standardized bus systems like CAN bus [1]. And today the FlexRay communications system has been successfully implemented and FlexRay standard is now a set of ISO standards, from ISO 17458-1 to 17458-5.

The FlexRay ISO standard contains conformance test specifications for FlexRay Protocol and Physical Layer. With the conformance tests released, semiconductor suppliers can submit their silicon solutions such as communication controllers and physical layer devices to the conformance test partners. Upon successful completion of the test, these devices will be qualified as conforming to the FlexRay ISO standard. For example, a FlexRay controller is going to be compliant to FlexRay communication controller requirements in ISO 17458-2 [2], it should pass all the test cases defined in the FlexRay communication controller test specification ISO 17458-3 [3]. Due to the complexity and professional degree of FlexRay conformance test, currently there are only few test partners such as TÜV Nord [4] and C\&S Group [5] all over the world. And this paper introduces a new test method designed especially for the FlexRay conformance tests to overcome the conformance testing difficulties.

\section{The FlexRay Protocol}

The FlexRay communication controller (abbreviated CC) is controlled by a host Micro-controller, to configure the parameters, sending and receiving communication data, as shown in the Fig. 1. The upper interface between CC and host can be in various forms, such as internal bus for integrated CC, or SPI and 
external bus for a standalone CC. The lower interface between CC and bus driver are defined only by three pins: TxD signal, RxD signal and TxEN signal [6], as shown in Fig. 2:

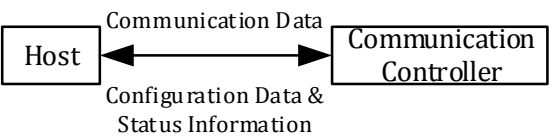

Fig. 1. Upper interface of CC.

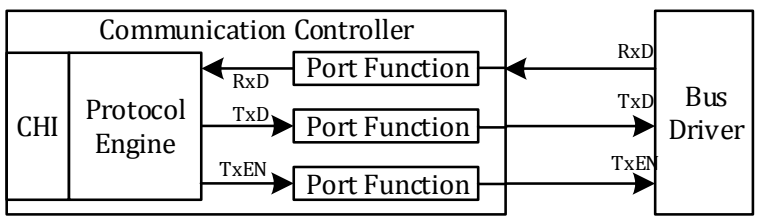

Fig. 2. Lower interface of CC.

\section{Test System Structure}

The test system structure follows ISO 17458-3 specification [3]. As according to ISO 9646 standard [7], the implementation under test (IUT) is the FlexRay CC, the upper tester (UT), the lower tester (LT) and the test supervisor (SV) are introduced. Fig. 3 describes the logical connection of these components.

UT plays the role of a user that makes use of the service provided by the IUT. LT plays the role of the peer entity of the IUT, which is connected to the FlexRay physical layer interface of the IUT. SV is connected to upper tester and lower tester at the same time, which controls the actions of LT and UT. SV is built as a test program running in computer. The LT is realized as a specific test tool in FPGA. The UT is a program implemented in the firmware of the micro-controller. In this paper, the Freescale 16-bit micro-controller MC9S12XF512 [8] with embedded FlexRay CC is chosen as an example for the implementation description.

Complete system block diagram of the current implementation is shown in Fig. 4. The UT and IUT are located in MCU, which is connected to PC through high-speed USB interface. LT is implemented in FPGA.

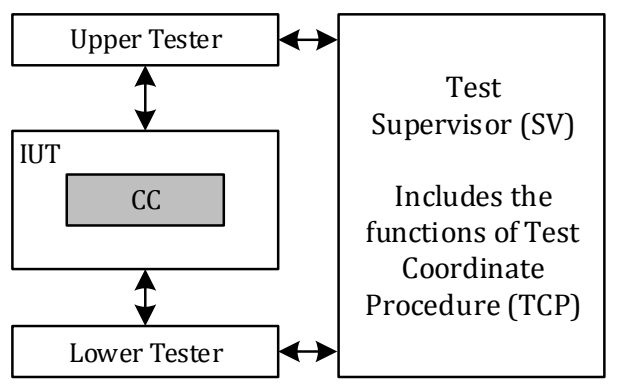

Fig. 3. FlexRay Protocol test structure according to ISO 964.

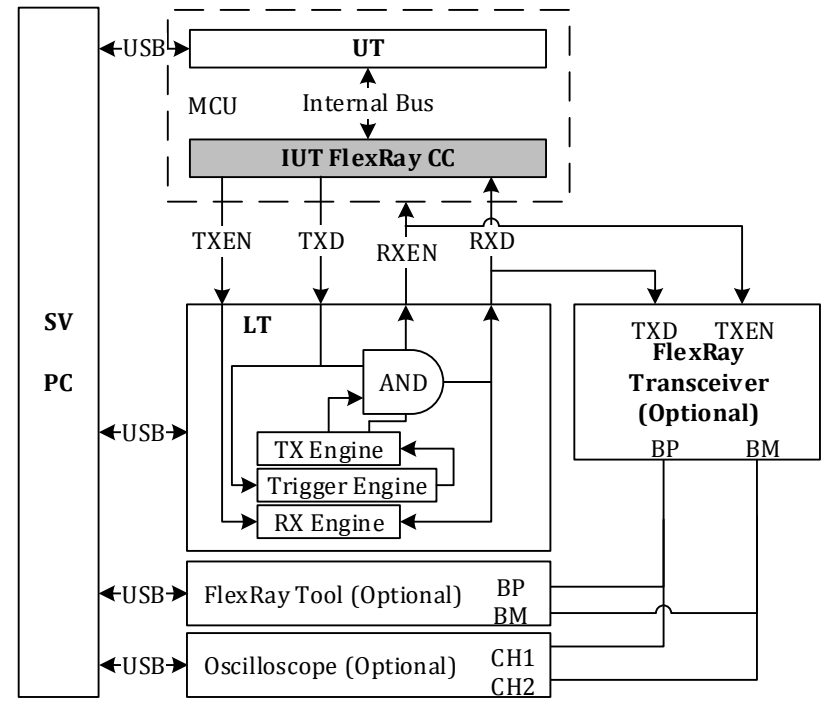

Fig. 4. Test system hardware components. 


\section{Implementation of LT}

In this chapter, hardware and software components of the proposed testing approach of LT are defined. Since FlexRay waveform can be decoded as raw bit stream, LT should include a waveform generator to simulate ordinary FlexRay frame with glitches, or a FlexRay frame with wrong CRC value [9].

\subsection{Functional System Blocks}

LT is designed to monitor and manipulate the three pins of CC: TXD, RXD and TXEN. The hardware components of LT are mainly located in a FPGA, as shown in the dashed box in Fig. 5, the Altera FPGA is used in the implementation, which is connected to PC with high-speed USB wire.

A NIOS II CPU is realized in the QSys system for overall control over the hardware components in the FPGA. The base frequency is fed by $50 \mathrm{M}$ Oscillator to generate $80 \mathrm{MHz}$ frequency for SDRAM module and the NIOS II CPU, other logics run on a generated PLL clock of $160 \mathrm{MHz}$ as according to ISO 17458-3 Table 2 [3].

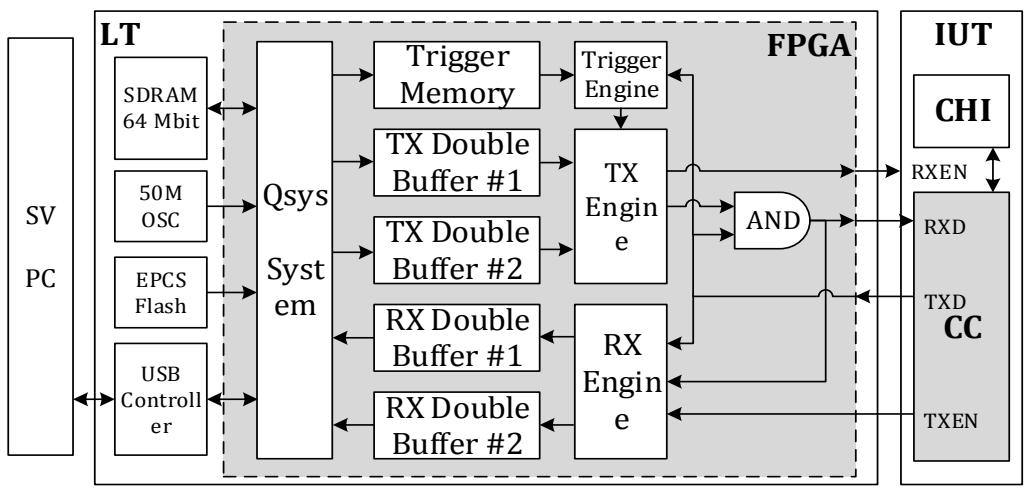

Fig. 5. Complete LT system block diagram.

\subsection{TX Engine}

TX Engine is responsible for transmitting arbitrary test sequences to $\mathrm{CC}$ as accurate as possible.

\subsubsection{Configuration}

To transmit arbitrary waveform at a frequency of $160 \mathrm{MHz}$, the TX engine should be able to read out the sequence quantum bits from internal memory while transmitting at the same time. Due to the significant delay exposed by external SDRAM, the internal On-Chip-Memory should be used. However, FPGA does not integrates large On-Chip-Memory. The solution is to adopt sequence coding and decoding technology.

\subsubsection{Sequence coding $\&$ decoding}

The upgraded configuration adopts a coding technology to represent a quantum bit by 16 memory bits including bit level and sequence length information. This scheme is shown in the Table 1 below:

Table 1. Coding Algorithm for TX Engine and RX Engine

\begin{tabular}{llll}
\hline \hline Bit 15 & Bit 14 & Bit 13 & Bit 12-0 \\
\hline TXEN Level & TXD Level & 16X Indicator & Sequence Length \\
\hline \hline
\end{tabular}

Bit 15 of the 16-bit configuration block is the voltage level of TXEN, and Bit 14 is TXD, Bit 12-0 contains 13 bits which specifies the time duration of TXD and TXEN, the maximum length is $2^{13}-1=8191$. Bit 13 is $16 \mathrm{X}$ multiple indicator, if this bit is 1 , the whole duration can be multiplied by 16 . This method effectively extends the waveform presentation ability. Take FlexRay wakeup symbol as an example, one wakeup symbol should be transmitted as $6 \mu \mathrm{s}$ low and $18 \mu \mathrm{s}$ high. With the help of this algorithm, the encoded sequence only takes 32 bits represented by 2 Words: 0x03C0 and 0x4B40, as described in Table 2 below: 
Table 2. FlexRay Wakeup Symbol Encoding Result

\begin{tabular}{|c|c|c|c|c|c|c|c|c|c|}
\hline $6 \mu \mathrm{s}$ low & Bit 15 & Bit 14 & Bit 13 & Bit $12-0$ & $18 \mu \mathrm{s}$ high & Bit 15 & Bit 14 & Bit 13 & Bit $12-0$ \\
\hline $0 \times 03 \mathrm{C} 0$ & 0 & 0 & 0 & $0 \times 3 \mathrm{CO}$ & $0 \times 4 B 40$ & 0 & 1 & 0 & $0 \times B 40$ \\
\hline
\end{tabular}

\subsection{RX Engine}

RX Engine plays the role of logic analyzer to sample the waveform from the TX pin, RX pin and TXEN pin of CC. As Recording FlexRay waveform directly into external SDRAM component in real-time requires complex algorithm and effort [10], to solve this problem, On-Chip-Memory should also be used. The RX Engine is responsible for encoding the input waveform into memory bits using the same method of TX Engine.

\subsection{Trigger Engine}

Trigger engine compares the waveform from the TXD of CC with the required waveform, if the waveform characteristics from CC match the desired waveform, a pulse is generated to trigger the TX Engine. The Trigger Engine implemented in FPGA has a state machine diagram as shown in Fig. 6.

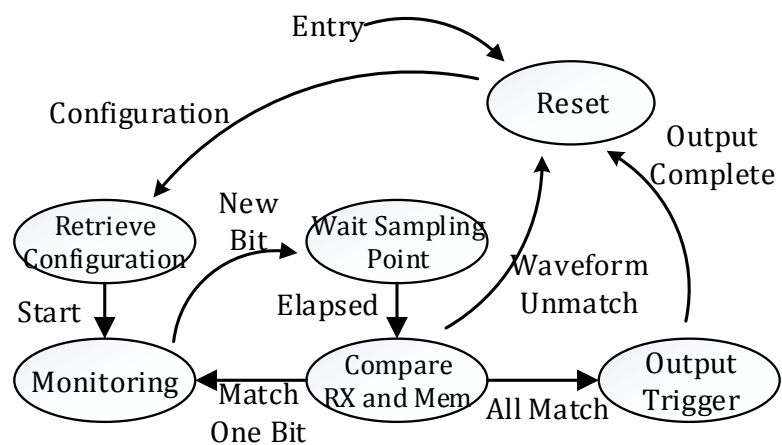

Fig. 6. Trigger engine state machine diagram.

\subsection{LT Configuration}

To control the activity of LT for the conformance test, specific commands should be supported by the CPU in the LT system. These commands are treated as Application Interface (API) from the standpoint of a tester while writing test scripts, the most important APIs are listed and described in Table 3.

Table 3. API List of LT

\begin{tabular}{ll}
\hline \hline API Name & Functional Description \\
\hline ConfigureTXEngine & Configure the TX Engine with arbitrary waveform sequences of 6.25ns precision \\
\hline ConfigureTriggerEngine & Configure the Trigger Engine with required sequence for outputting trigger pulse \\
\hline StartTXEngine & Trigger the TX Engine transmit the configured sequences \\
\hline StartTriggerEngine & Start the Trigger Engine to listen to the TXD of CC \\
\hline StopTriggerEngine & Stop the Trigger Engine running \\
\hline RetrieveRXEngine & Retrieve the recorded waveform sequences from RX Engine with 6.25ns precision \\
\hline
\end{tabular}

All the commands are executed synchronously during the conformance test, for example, if the TX Engine will be started only if it is configured first, and only if the configuration is successful, the scripts should be:

$$
\text { if (!ConfigureTXEngine(/* parameters */)) return; }
$$

if (!StartTXEngine()) return; 


\section{Implementation of UT}

In the current implementation, the UT is partly distributed into the host MCU MC9S12XF512. The function block of UT is shown in Fig. 7. Each important function block is described in the following chapters.

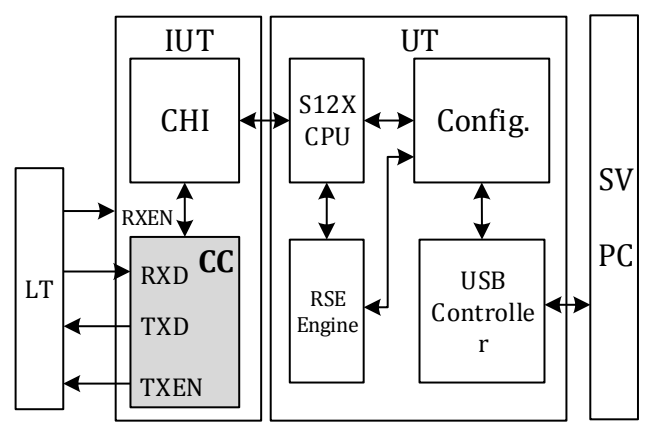

Fig. 7. UT functional block structure.

\subsection{UT Configuration Module}

Configuring the registers and buffers of CC is the major task for UT, which is handled in the UT Configuration Module. A set of APIs are also available for the testers for dedicated configuration. Due to the fact that FlexRay CC requires hundreds of register values in the configuration, which vary among different test cases. A solution is to build a User Interface which supports basic configurations, preambles, modifications, variants, as shown in Fig. 8.

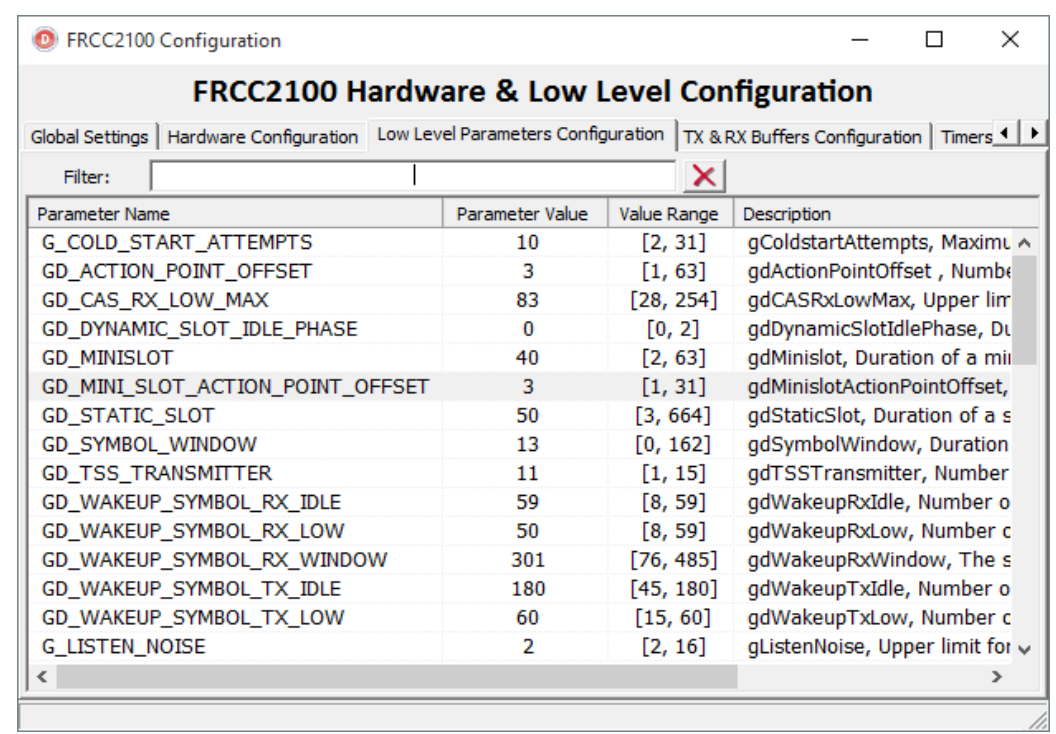

Fig. 8. Dedicated CC configuration user interface.

\subsection{FlexRay Frame Simulation}

Another important part during the conformance test is FlexRay payload data transmission and reception, the payload contents for transmission of the IUT are defined in the test specification, however, the payload data changes in test cases and even cycles. To ease the desired payload data transmission from CC to LT, a universal hardware script execution engine (RSE Engine) is developed for manipulating the real-time simulation logic of the IUT's transmission payloads [11]. With the help of the good real-time performance of RSE engine, the payload from CC can be configured precisely in each cycle. 


\section{Implementation of SV and Test System}

The test system is made up of Test Engine, Test Scripts, Configuration Modules and Basic Modules, as shown in Fig. 9. The Test Engine in the center controls all the external instruments and devices including UT and LT. This test method is open architecture and supports multi-automotive networks [12]. Test scripts, test configurations and Basic Modules are loaded by the Test Engine before execution of each test case.

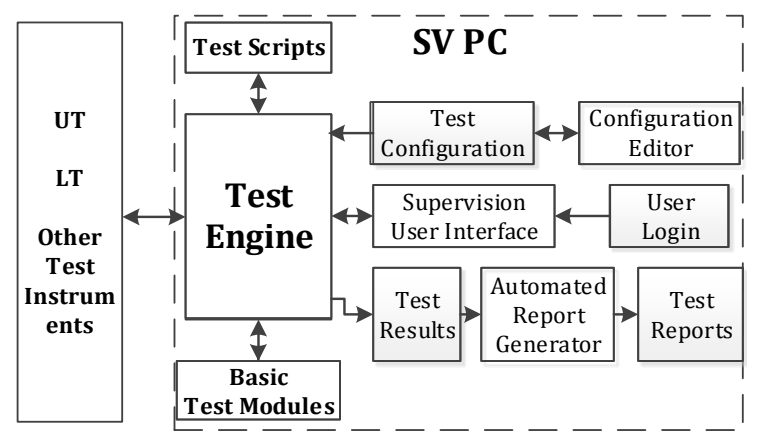

Fig. 9. Function blocks of SV and test system on PC.

\subsection{Test Engine Design}

The most important feature of the test engine is to control every device independently at the same time so as to coordinate the operations performed by LT and UT. It is achieved by creating a standalone thread for execution of all basic modules. A basic module stands for an abstraction of one test device, such as UT.

The Test logic according to ISO 17458-3 is implemented in Test Scripts in C language, which is represented as a set of source and header files. A C compiler is used to compile the scripts source into binary file that can be invoked by the test engine. Test parameters of FlexRay CC are imported by the Test Engine and assigned to global variables of the script program. APIs from the basic modules are then invoked by the scripts to execute the steps required by the CC test specification.

\subsection{Test Procedure}

The test procedure defined in ISO 17458-3 should be followed strictly. The procedure is shown in Fig. 10, which includes three main loops in each test case:

- Instances: Three instances are available: pChannels $=\mathrm{A}, \mathrm{pChannels}=\mathrm{B}$ and pChannels $=\mathrm{A} \& \mathrm{~B}$

- Modifications: Modification tables that is going to replace the value defined in the basic configurations

- Variants: Execution dependency, each variant should be executed separately

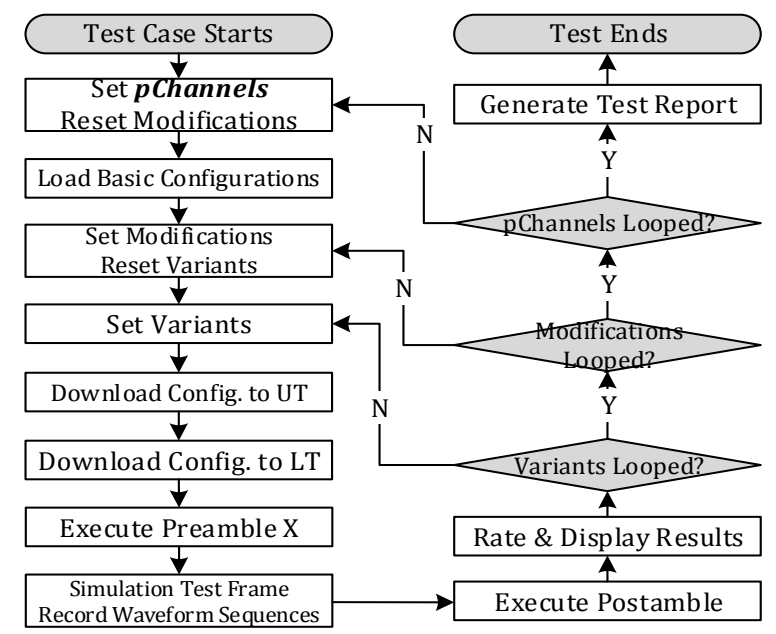

Fig. 10. Procedure of a test case according to ISO 17458-3. 
If there are 3 instances, 2 modifications and 3 variants, the total execution count should be $3 * 2 * 3=18$. All the parameter loading and loop switching are handled by the basic module DLL module.

\subsection{Test System User Interface}

The main part of Test System interface is shown in Fig. 11. The tree-view on the left side collects all the test cases from ISO 17458-3. The selected test cases can be executed by clicking the "Start Test" button.

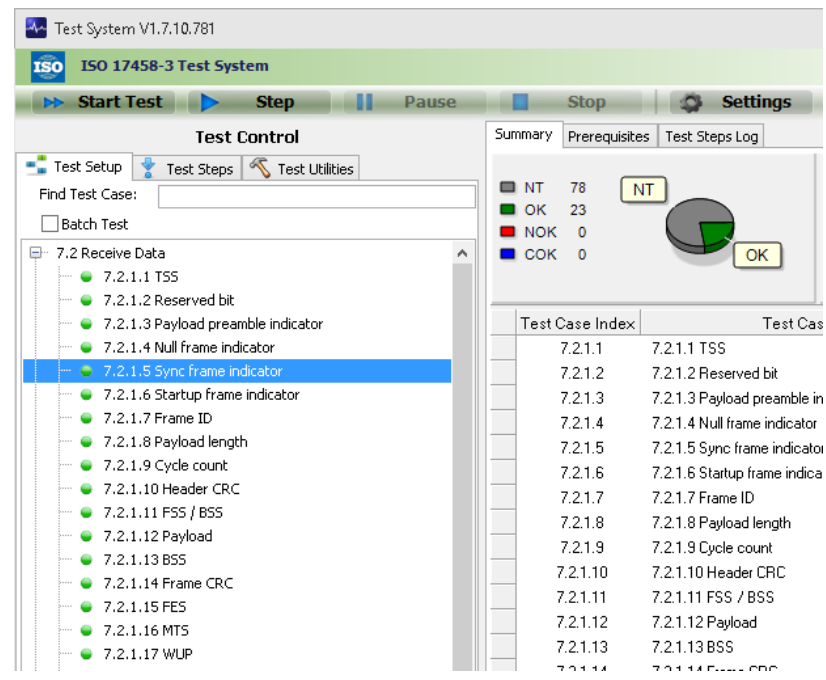

Fig. 11. Test system main interface.

\section{Results and Analysis}

One of a test case from ISO 17458-3 is described to verify the current test solution. The selected test case is 7.2.1.7 - Frame ID. The purpose of this test case is to verify that the CC does not update frame contents data upon reception of invalid frames. Apart from the test procedure described above, the remaining steps inside the execution include the following actions:

In the cycles after Preamble I, LT simulates the specified ID with correct/incorrect CRC, CC should set the corresponding registers as described in the test specification: Frame ID register; content error flag/indicator; valid frame flag/indicator; contents data value.

During the execution, the UT and LT are first configured by the test system, and the Preamble I is executed as prerequisite for this test case. The CC has entered normal active state in Preamble I, the overview of the communication cycles monitored by RX Engine is shown in Fig. 12 below, from cycle 0 to cycle 13 . High level is logic 1 and low level is logic 0 .

The recorded waveform contains minimum quantum bits separated by $6.25 \mathrm{~ns}$ at a $160 \mathrm{MHz}$ sample rate, after the waveform is zoomed in, the detailed bit time can be analyzed, Fig. 13 displays the LT's normal frame in slot 1 and CC's normal frame in slot 2 in Cycle 8. The frame content is analyzed by the FlexRay test module of the test system running in PC. The corresponding register value and frame content are retrieved by UT.

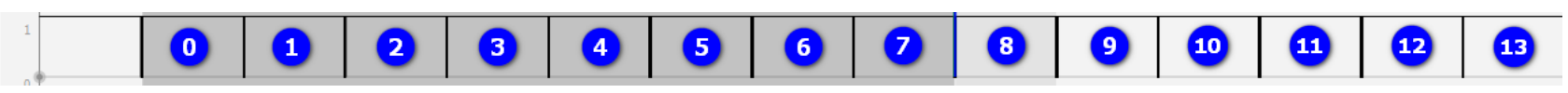

Fig. 12. Overview of all the communication cycles starting from Cycle 0 to Cycle 11.

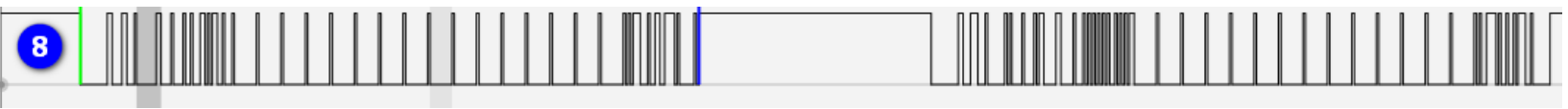

Fig. 13. The frame content from LT and CC during Cycle 8. 
After waveform and register comparison, the test result of this test case is shown in Table 4:

Table 4. Test Result Summary for Test Case 7.2.1.7

\begin{tabular}{lllll}
\hline \hline Comm. Cycle & Frame ID & Content Error Flag & Valid Frame Flag & Frame Content \\
\hline Cycle 8 & 1 & False & True & Updated \\
\hline Cycle 9 & 0 & True & False & Not updated \\
\hline Cycle 10 & 2 & True & False & Not updated \\
\hline Cycle 11 & 1 & False & True & Updated \\
\hline
\end{tabular}

All the test instances, test modifications are looped by the test engine and the result shows that the execution of each step is correct according to ISO 17458-3.

\section{Conclusion}

This paper presents a new test method for the FlexRay data-link layer Conformance Test according to ISO 17458-3. Test devices including LT, UT, SV and Test System are built against the ISO 9646 standard Conformance Test method. This system stands for a low-cost, reliable and high-performance test solution for the Conformance Test of FlexRay communication controller. Due to the open architecture of the system components, this solution can be extended for other dedicated Conformance Test systems such as CAN-FD, CAN and LIN bus in automotive communications system.

\section{References}

[1] ISO, ISO 17458-1. (2013). Road vehicles - FlexRay communications system — Part 1: General information and use case definition. International Organization for Standardization.

[2] ISO, ISO 17458-2. (2013). Road vehicles - FlexRay communications system — Part 2: Data link layer specification. International Organization for Standardization.

[3] ISO, ISO 17458-3. (2013). Road vehicles - FlexRay communications system — Part 3: Data link layer conformance test specification. International Organization for Standardization.

[4] Electonics and IT in the Vehicle, with intelligent programs for clever solutions. From http://www.tuv-nord.com/en/systems-components/electronics-it-4923.htm

[5] We offer: FlexRay Physical Layer conformance tests for your bus drivers or active stars. From http://www.cs-group.de/testing/conformance-tests/flexray.html

[6] ISO, ISO 17458-4. (2013). Road vehicles — FlexRay communications system — Part 4: Electrical physical layer specification. International Organization for Standardization.

[7] ISO, ISO 9646. (1991). Information Technology, Open Systems Interconnection, Conformance Testing Methodology and Framework. International Standard IS-9646. ISO, Geneve.

[8] Freescale. (November 10, 2010). MC9S12XF512 Reference Manual. Rev.1.20.

[9] Milbredt P., Steininger, A., \& Horauer, M. (2008). Automated testing of FlexRay clusters for system inconsistencies in automotive networks. Proceedings of the 4th IEEE International Symposium on Electronic Design, Test \& Applications (DELTA 2008) (pp. 533-538). Hong Kong.

[10] Horauer, M., Rothensteiner, F., Zauner, M., Armengaud, E., Steininger, A., Friedl, H., \& Pallierer, R. (2004). An FPGA based SoC design for testing embedded automotive communication systems employing the FlexRay protocol. Proceedings of the Austrochip 2004 Conference (pp. 119-123). Villach, Austria.

[11] Chu. L., Feng. L., Yunge, Q. (2015). Real-time simulation engine implementation in automotive FlexRay communication. International Journal of Computer and Communication Engineering, 4(4), 246-255.

[12] Feng, L, \& Chu, L. (2013). Research on can bus automated test environment. Journal of Theoretical \& Applied Information Technology, 47(3), 1271-1276. 


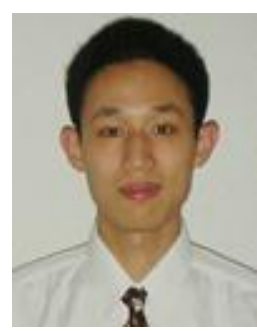

Chu Liu was born in Fujian, China in July 1985. He received his master's degree in automotive electronics in March 2011 at Clean Energy Automotive Engineering Center, College of Automotive Engineering, Tongji University, Shanghai, China.

$\mathrm{He}$ is now a PhD research student in Clean Energy Automotive Engineering Center in Tongji University in Shanghai, China. His research interests include automotive network communication, vehicle to vehicle communication and intelligent vehicle.

Dr. Chu Liu has joined in the "Tongji-Freescale" joint laboratory and "Tongji-ihr" joint laboratory and he is doing research in the automotive network field for many years.

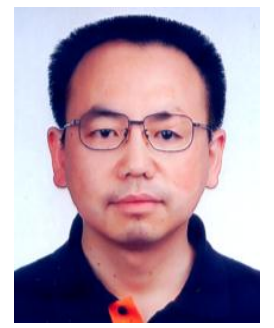

Feng Luo was born in Shanxi, China on September 1969. He received his PhD degree in aircraft control engineering from Northwestern Polytechnical University in 2000, Shanxi, China. From 2000 to 2002, he did his postdoctoral research work in the Department of Automotive Engineering of Tsinghua University in Beijing, China.

He came to Tongji University in 2002, he is now a professor in automotive engineering study of Tongji University. His research interests include automotive networks, automotive electronic system control, vehicle to vehicle communication.

Prof. Feng Luo is now the director of "Tongji-Freescale" joint laboratory and "Tongji-ihr" joint laboratory. His automotive development group has been participating in numerous national and international research projects in these areas for more than 10 years. 